\title{
Positioning Errors Predicting Method of Strapdown Inertial Navigation Systems Based on PSO-SVM
}

\author{
Xunyuan Yin, Yingbo Sun, and Changhong Wang \\ Space Control and Inertial Technology Research Center, Harbin Institute of Technology, Harbin 150001, China \\ Correspondence should be addressed to Changhong Wang; cwang@hit.edu.cn
}

Received 13 July 2013; Revised 27 July 2013; Accepted 27 July 2013

Academic Editor: Hamid Reza Karimi

Copyright ( 2013 Xunyuan Yin et al. This is an open access article distributed under the Creative Commons Attribution License, which permits unrestricted use, distribution, and reproduction in any medium, provided the original work is properly cited.

\begin{abstract}
The strapdown inertial navigation systems (SINS) have been widely used for many vehicles, such as commercial airplanes, Unmanned Aerial Vehicles (UAVs), and other types of aircrafts. In order to evaluate the navigation errors precisely and efficiently, a prediction method based on support vector machine (SVM) is proposed for positioning error assessment. Firstly, SINS error models that are used for error calculation are established considering several error resources with respect to inertial units. Secondly, flight paths for simulation are designed. Thirdly, the $\varepsilon$-SVR based prediction method is proposed to predict the positioning errors of navigation systems, and particle swarm optimization (PSO) is used for the SVM parameters optimization. Finally, 600 sets of error parameters of SINS are utilized to train the SVM model, which is used for the performance prediction of new navigation systems. By comparing the predicting results with the real errors, the latitudinal predicting accuracy is $92.73 \%$, while the longitudinal predicting accuracy is $91.64 \%$, and PSO is effective to increase the prediction accuracy compared with traditional SVM with fixed parameters. This method is also demonstrated to be effective for error prediction for an entire flight process. Moreover, the prediction method can save $75 \%$ of calculation time compared with analyses based on error models.
\end{abstract}

\section{Introduction}

Strapdown inertial navigation systems have been widely utilized in a wide range of fields, such as the navigation of airplanes, ships, and vehicles and the guidance of missiles [1]. Although the positioning accuracy of strapdown systems is lower than that of the platform inertial navigation systems, the strapdown systems have several advantages that cannot be found in platform systems. They have low cost, low weight, small volume, good reliability, and simple mechanical structures [2]. So far, almost all the civil aviation airplanes manufactured by Boeing and Airbus are equipped with LTN92 or LTN-101 laser SINS $[3,4]$. Other types of SINS that consist of different kinds of gyroscopes and accelerometers are used for the navigation of vehicles which require medium or low accuracy [5].

Navigation accuracy is the main factor that is used to access the performance of SINS [6]. To help the vehicles to complete the flight task or arrive at a desired destination, the strapdown systems which can meet the navigation requirements should be selected [7]. If an effective method that could be established to predict the velocity errors and positioning errors by assessing error parameters, a good deal of time for error analyses would be saved and the aircrafts would be more likely to accomplish the initial tasks $[8,9]$. Currently, some researchers have placed importance on the error analyses and error compensation of SINS [10]. A methodology based on the theory of artificial neural networks has been put up to predict the positioning errors caused by the drift error of each singe axis of gyroscope [11]. But research that stresses predicting the performance of SINS by error sources can hardly be found.

Nowadays, several methodologies have been reported for the classification or regression in various fields. Artificial neural networks (ANNs) have been pervasively adopted and are able to achieve acceptable results in many applications. Chen developed an ANN-based model, which is called Evolutionary Fuzzy Neural Inference Model, to predict Estimate 
at Completion (EAC) [12]. However, it has disadvantages to address the proposed problem since it is shown by simulation that the predicting accuracy might be low due to a local minimization problem. As a consequence, it is not guaranteed for all the models to converge to optimal solutions [13]. Besides, ANN is also vulnerable to the selection of network structure, and it has a high computational expense in terms of training process. Extended Kalman filter (EKF) approach, which is also popular in industrial applications, is able to maintain relatively high accuracy in terms of estimation, but EKF estimator often requires high cost due to high computational complexity [14]. Fuzzy logic method is also reported to be available for estimation. For more details on state estimation and, on filtering approaches for complex systems the reader can be referred to [15-19].

Support vector machine (SVM) is an effective mathematical method in prediction, and this technique has greatly developed in the past decades. SVM, which is based on structural risk minimization principle [20], is adopted by researchers to address classification and regression problems [21]. So far, it has been successfully used in a variety of fields. For instance, it is used for noise estimation and the prediction of air passengers [22,23], it is also utilized for image analyses, biomedicine, and bioinformatics as estimator tools.

Inertial navigation systems are sophisticated nonlinear systems [24]. Therefore, it is unrealistic to estimate the performance of the navigation systems by the analyses of the models. In order to predict the navigation errors by error coefficients, a method based on support vector machine is proposed in this paper. Support vector machine, unlike other traditional methods, is relatively effective in terms of combating nonlinear situations. It is more robust as an estimator than least-square based method because it is insensitive to small changes [25]. Firstly, the error model of SINS is established. Secondly, a series of flight paths that meet the characteristics of real trajectories are designed. Then, 300 sets of random error parameters which obey Gaussian distribution are generated, and results of system errors are obtained by simulation. These parameters are used to train the model of SVM. Finally, the trained model is used to predict the system errors of SINS with different error coefficients. This method is tested to be effective and efficient by comparing simulation results with the actual navigation errors.

The remainder of this paper is organized as follows. Error model of SINS is given, and fight path for simulation is designed in Section 2. In Section 3, SVM-based navigation error estimation method is proposed. Simulation verification of proposed prediction method is given, and error prediction of an entire flight path is completed in Section 4. Finally, Section 5 gives the conclusions.

\section{Error Model of SINS}

Error equations, in terms of velocity errors, attitude errors and position errors, should be established in order to analyze the navigation errors caused by strapdown inertial navigation systems [2].
2.1. Attitude Error Equations. The attitude errors of the aircraft are represented by $\delta \alpha, \delta \beta$, and $\delta \gamma$, respectively, as follows:

$$
\begin{aligned}
\delta \dot{\alpha}= & -\frac{\delta V_{N}}{R}+\left(\frac{V_{E}}{R} \tan \varphi+\omega_{i e} \sin \varphi\right) \delta \beta \\
& -\left(\frac{V_{E}}{R}+\omega_{i e} \cos \varphi\right)+\varepsilon_{E}, \\
\delta \dot{\beta}= & \frac{\delta V_{E}}{R}-\left(\frac{V_{E}}{R}+\omega_{i e} \sin \varphi\right) \delta \alpha \\
& -\omega_{i e} \sin \varphi \delta \varphi-\frac{V_{N}}{R} \delta \gamma+\varepsilon_{N}, \\
\delta \dot{\gamma}= & \frac{\delta V_{E}}{R} \tan \varphi+\left(\frac{V_{E}}{R} \sec ^{2} \varphi+\omega_{i e} \cos \varphi\right) \delta \varphi \\
& +\left(\frac{V_{E}}{R}+\omega_{i e} \cos \varphi\right) \delta \alpha+\frac{V_{N}}{R} \delta \beta+\varepsilon_{\xi},
\end{aligned}
$$

where $\varepsilon_{N}, \varepsilon_{E}$, and $\varepsilon_{\xi}$ represent the gyroscope drift errors of three axes.

2.2. Velocity Error Equations. With respect to the navigation system which is analyzed in this passage, some error sources, such as the ones related to acceleration, can be ignored when the coefficients are relatively small. Therefore, velocity error equations are established in the following:

$$
\begin{aligned}
\delta \dot{V}_{E}= & \left(\frac{V_{E}}{R} \tan \varphi-\frac{V_{\xi}}{R}\right) \delta V_{E}+\left(\frac{V_{E}}{R} \tan \varphi+2 \omega_{i e} \sin \varphi\right) \delta V_{N} \\
& -\left(\frac{V_{E}}{R}+2 \omega_{i e} \cos \varphi\right) \delta V_{\xi} \\
& +\left(\frac{V_{E} V_{N}}{R} \sec ^{2} \varphi+2 \omega_{i e} \cos \varphi V_{N}+2 \omega_{i e} \sin \varphi V_{\xi}\right) \delta \varphi \\
& +A_{N} \gamma-A_{\xi} \beta+\Delta A_{E}, \\
\delta \dot{V}_{N}= & -2\left(\frac{V_{E}}{R} \tan \varphi+\omega_{i e} \sin \varphi\right) \delta V_{E}-\frac{V_{\xi}}{R} \delta V_{N}-\frac{V_{N}}{R} \delta V_{\xi} \\
& -\left(\frac{V_{E}}{R} \sec ^{2} \varphi+2 \omega_{i e} \cos \varphi\right) V_{E} \delta \varphi \\
& +A_{\xi} \alpha-A_{E} \gamma+\Delta A_{N}, \\
\delta \dot{V}_{\xi}= & 2\left(\frac{V_{E}}{R}+\omega_{i e} \cos \varphi\right) \delta V_{E}+2 \frac{V_{N}}{R} \delta V_{N}-2 V_{E} \omega_{i e} \sin \varphi \delta \varphi \\
& +A_{E} \beta-A_{N} \alpha+\Delta A_{\xi},
\end{aligned}
$$

where $\Delta A_{E}, \Delta A_{N}$, and $\Delta A_{\xi}$ represent the accelerometer $\mathrm{drift}$ errors of three axes.

2.3. Positioning Error Equations. The positioning errors are the main factors that are considered when evaluating the performance of different kinds of aircrafts, such as airplanes and 


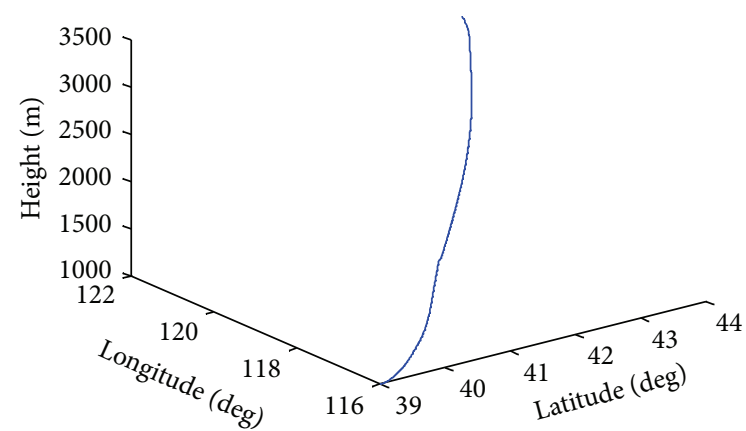

FIGURE 1: Flight path for analyses.

UAVs. Therefore, the latitudinal and longitudinal errors are important factors for the assessment of navigation systems:

$$
\begin{gathered}
\delta \dot{L}=\frac{\delta V_{N}}{R}-\delta h \frac{V_{N}}{R^{2}}, \\
\delta \dot{\lambda}=\left(\frac{\delta V_{E}}{R}+\delta L \frac{V_{N}}{R^{2}} \tan L\right) \sec L-\delta h \frac{V_{E} \sec L}{R^{2}}, \\
\delta \dot{h}=\delta V_{U} .
\end{gathered}
$$

2.4. Flight Path Design. According to several papers that focus on error analysis of inertial navigation systems, the research is mainly based on some simple flight paths, such as uniform linear motion or uniform turning motion. However, these flight paths cannot involve all the flight modes. As a consequence, the results of these simulations or analyses may not represent the real performance of the aircrafts that are equipped with SINS.

Therefore, it is necessary to design some flight paths that not only include the characteristics of real paths of aircrafts but are also able to ensure for each error source to be stimulated. As shown in Figure 1, a typical flight path for simulation is designed.

\section{Navigation Error Prediction}

It is unrealistic to use error equations to analyze the performance of SINS, especially when there is a large variety of systems that should be tested in short term, as it will cost a good deal of time to solve the differential error equations.

In order to avoid complicated calculations, support vector machine with strong generalization ability is utilized to predict system errors by assessing each single error source. However, a noticeable problem is that the navigation errors are time varying and closely associated with the flight paths. Therefore, characteristic vectors related to certain flight paths and ultimate positioning errors should be established to accomplish the prediction, because positioning errors are the most important data for navigation systems.

3.1. Support Vector Regression. Support vector machine (SVM), a method closely associated with optimization algorithms, is an effective methodology to address data processing problems [12]. It is demonstrated to be eligible to overcome the traditional obstacles with respect to multidimensional problems and over learning. So far, SVM is widely used in many fields, such as biological information, voice recognition, failure identification, and prognostics.

SVM consists of support vector classification and support vector regression. To solve problems with respect to prediction, support vector regression method can be used [21]. Since the problem is nonlinear, a transform $x=\phi(x)$ should be introduced. By using a nonlinear mapping that maps the sample data into a high dimensional space: $\phi$ : $R^{n} \rightarrow H$, linear regression method can be conducted in the high-dimensional space $H$ to accomplish the nonlinear prediction.

A training set is given as

$$
T=\left\{\left(x_{1}, y_{1}\right),\left(x_{2}, y_{2}\right), \ldots,\left(x_{l}, y_{l}\right)\right\} \in\left(R^{n} \times y\right)^{l} .
$$

Due to the varying characteristics of the error parameters of inertial navigation systems, a leading problem that should be overcome is that all the significant parameters with respect to positioning errors should be preprocessed as characteristic quantities for estimation, which is considered to be effective to improve the estimation accuracy [26]. Therefore, 15 error parameters that have considerable impacts on position errors of SINS are considered for the model training. In (4), $x_{i}$ represent the error sources of SINS, which are the zero bias errors, random walk errors of gyros, zero random walk of accelerometers, random walk errors of accelerometers, and scale factor errors of gyros. $y_{i}$ represent navigation errors, which are latitudinal and longitudinal positioning errors of the training models. Compared with ANNs, SVM has a drawback that it can only generate one output, while the ANNs are able to generate multiple outputs [27]. Consequently, two separate training processes should be separately conducted for latitudinal and longitudinal errors. Specifically, the error coefficients and positioning errors of the 600 sets of navigation systems are used to train the SVM model. Then, a suitable kernel function is selected as radial Basis Function (RBF). As there are many kernel functions available for analyses $[28,29], \mathrm{RBF}$ is demonstrated to be effective for this problem by contrasting with other kernel functions:

$$
K\left(x_{i}, x_{j}\right)=\exp \left(-\frac{\left\|x_{i}-x_{j}\right\|^{2}}{2 \sigma^{2}}\right) .
$$

In the next step, following convex quadratic programming, problem (5) is resolved to obtain that $\bar{\alpha}^{(*)}=$ $\left(\bar{\alpha}_{1}, \bar{\alpha}_{1}^{*}, \ldots, \bar{\alpha}_{l} \bar{\alpha}_{l}^{*}\right)^{T}$ as follows:

$$
\begin{array}{r}
\min \frac{1}{2} \sum_{i, j=1}^{l}\left(\alpha_{i}^{*}-\alpha_{i}\right)\left(\alpha_{i}^{*}-\alpha_{i}\right) K\left(x_{i}, x_{i}\right) \\
+\varepsilon \sum_{i=1}^{l}\left(\alpha_{i}^{*}+\alpha_{i}\right)-\sum_{i=1}^{l} y_{i}\left(\alpha_{i}^{*}-\alpha_{i}\right) .
\end{array}
$$

If $\bar{\alpha}_{i}$ is picked, and then $\bar{b}=y_{i}-\sum_{i=1}^{l}\left(\bar{\alpha}_{i}^{*}-\bar{\alpha}_{i}\right) K+\varepsilon$, if $\bar{\alpha}_{k}^{*}$ is picked, then $\bar{b}=y_{k}-\sum_{i=1}^{l}\left(\bar{\alpha}_{i}^{*}-\bar{\alpha}_{i}\right) K-\varepsilon$. 
Decision function is established as

$$
y=g(x)=\sum_{i=1}^{l}\left(\bar{\alpha}_{i}^{*}-\bar{\alpha}_{i}\right) K\left(x_{i}, x\right)=\bar{b}
$$

3.2. PSO-Based Optimal SVR Parameters Selection. Important factors of SVM are the constant $C$, the accuracy parameter $\varepsilon$, and the kernel function. As the kernel function has been selected as RBF, appropriate $C$ and $\varepsilon$ should be selected in order to increase the estimation accuracy. Since it may take an extremely long time to seek the best parameters and desired results may not be achieved by simply making different tests, an efficient method called particle swarm algorithm is adopted for the optimization.

Particle swarm algorithm (PSO) is an algorithm that is inspired by birds' foraging behavior and widely used to address optimization problems [30-32]. The PSO algorithm is introduced to optimize the parameters of $\varepsilon$ and $C$. The PSO is initialized with random particles, and then it works to find optimal parameters by iterative methods. Practically, the initial parameters of $\varepsilon$ and $C$ should be given, and then the optimal values will be generated by calculation.

\section{Error Analyses for SINS}

Before predicting the performance of certain inertial navigation systems, error analyses for SINS, which are indispensable for the process of error prediction, should be conducted. As for the strapdown navigation systems studied in this paper, the drift errors of the gyroscopes, the white noise of the gyroscopes, and the drift errors of accelerometers are considered, whereas the error coefficients related to acceleration and the coefficients related to quadratic acceleration are ignored, since such coefficients are comparatively small.

Two criteria should be obeyed for the selection of training data

(1) The training data should be not the same as the data for test. If there were no differences between the training data and testing data, the prediction accuracy would be relatively high but biased.

(2) The dimension of the inputs should be increased if possible. If the training data had a high dimension, more useful characteristics could be used for model training.

(3) The training data should represent different systems with vastly different error coefficients. With respect to this criterion, suitable standard deviation for error sources should be assigned.

The error sources are assigned by the given parameters which are listed in Table 1 . The navigation system is affected by multiple error sources when it performs a navigation task, so it is necessary to assign all the error parameters that are considered in this navigation system in order to make the results adaptive for real flight situations. To make preparation for the performance prediction of SINS, it is sensible to carry out error analyses for 600 sets with different
TABLE 1: The expected value and standard deviation of error sources.

\begin{tabular}{lcc}
\hline Error sources & $\begin{array}{c}\text { Expected } \\
\text { value }\end{array}$ & $\begin{array}{c}\text { Standard } \\
\text { deviation }\end{array}$ \\
\hline Zero bias errors of gyros & $0.01^{\circ} / \mathrm{h}$ & $0.006^{\circ} / \mathrm{h}$ \\
Random walk errors of gyros & $0.001^{\circ} / \sqrt{\mathrm{h}}$ & $0.0068^{\circ} / \sqrt{\mathrm{h}}$ \\
Accelerometer zero bias errors & $3 \mu \mathrm{g}$ & $0.5 \mu \mathrm{g}$ \\
Accelerometer random walk errors & $3 \mu \mathrm{g} / \sqrt{\mathrm{Hz}}$ & $5 \mu \mathrm{g} / \sqrt{\mathrm{Hz}}$ \\
Scale factor errors of gyros & $10 \mathrm{ppm}$ & 0 \\
\hline
\end{tabular}

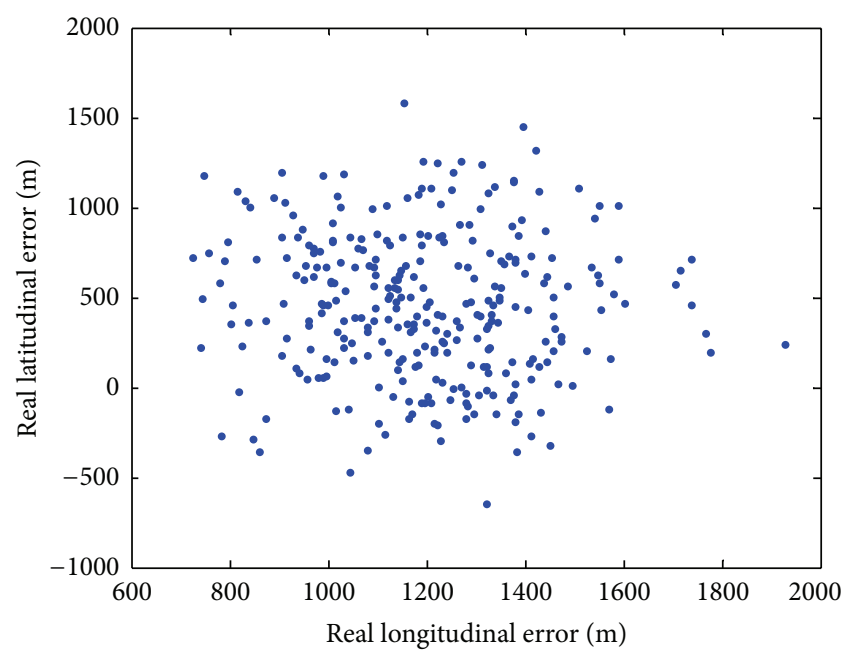

FIGURE 2: Real distributing points of positioning errors.

error parameters. The assignment of different error sources abides by the Gaussian distribution. Calculating with the system error functions, the 600 sets of navigation errors of the navigation systems with different error coefficients are achieved.

4.1. Simulation Verification of Prediction Method. The error coefficients and the positioning error parameters of the 600 sets of SINS are transformed into characteristic values, which are used to train the SVM model. Other 300 sets of error coefficients are randomly generated, and the corresponding system errors are calculated by error equations. Then, the accuracy parameter is initially given as $\varepsilon=0.5$, and the penalty parameter is given as $C=60$. PSO is used to generate optimal parameters of $\varepsilon=0.0104$ and $C=49.12$.

Both original SVM with fixed parameters and SVM model with optimal parameters generated by PSO are used in order to predict the positioning errors. The real distribution points of positioning errors are shown in Figure 2, while the predicting distribution points of positioning error are shown in Figure 3.

The prediction errors in terms of latitude and longitude which are predicted by SVM model with optimal parameters generated by PSO are shown in Figure 4.

By comparing the results, the predicting results are relatively satisfying. Specifically, the average north error is $34.3 \mathrm{~m}$, while the expected value of north error by prediction is $455.8 \mathrm{~m}$. The standard deviation of north error by prediction 


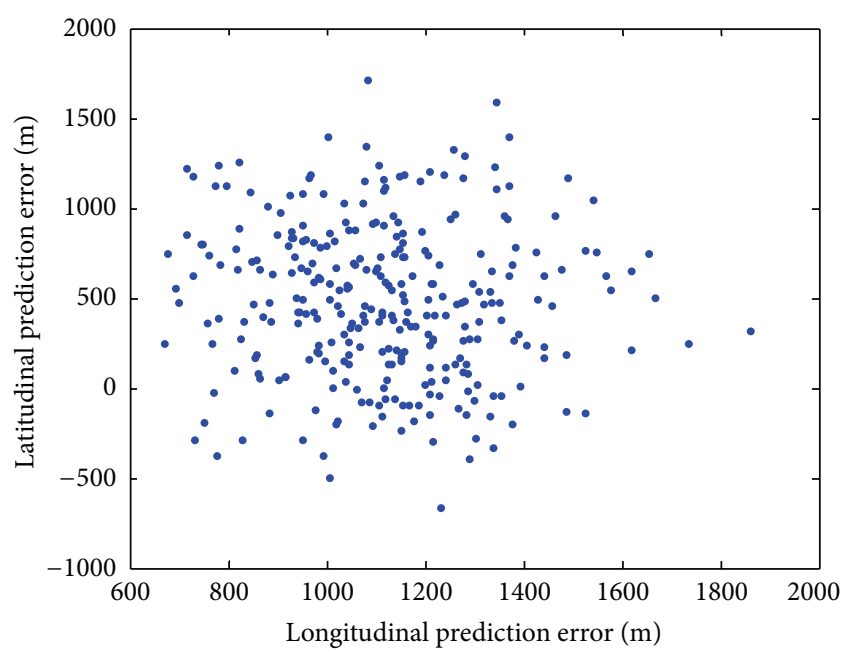

FIgURE 3: Prediction distributing points of positioning errors.

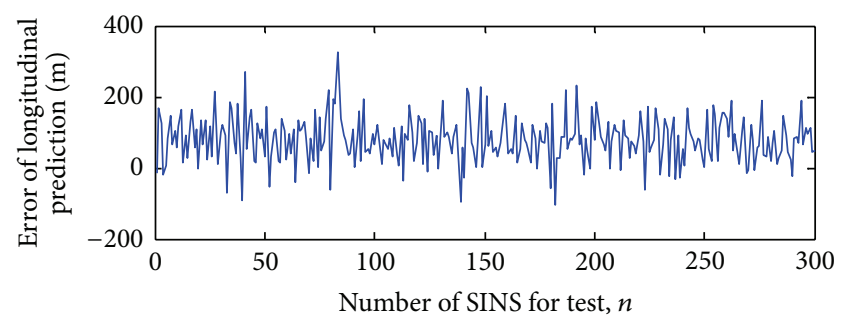

(a)

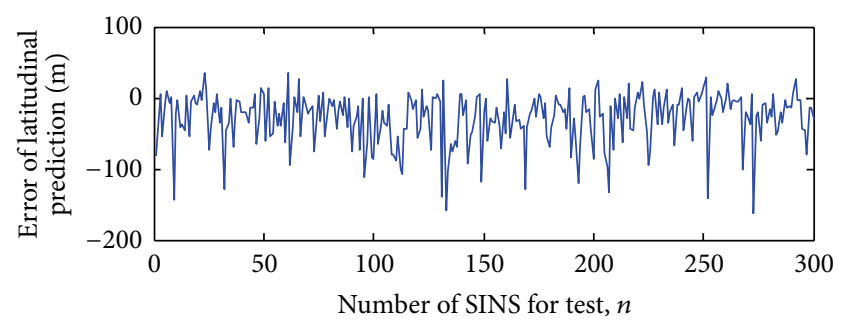

(b)

FIgURE 4: Prediction errors generated by SVM.

is $45.7 \mathrm{~m}$. The average east error is $96.4 \mathrm{~m}$, while the expected value of east error by prediction is $1194.6 \mathrm{~m}$. The standard deviation of east error by prediction is $103.7 \mathrm{~m}$.

The predicting accuracy is defined as $P$, which is accessed with (7). $e_{p}$ represents the predicting error, and $e_{r}$ represents the real error calculated by system error equations. Letter $n$ represents the number of navigation systems for test:

$$
P=1-\sum_{i=1}^{n}\left|\frac{e_{p i}-e_{r i}}{e_{r i}}\right| \times \frac{1}{n} .
$$

After calculation, the predicting accuracy is seen in Table 2, the latitudinal predicting accuracy is $92.73 \%$, while the longitudinal predicting accuracy is $91.64 \%$. The accuracy of PSO-based method is noticeably higher than that of original SVM. There is also a substantial decrease in the time which is spent assessing the SINS performance. Specifically,
TABLE 2: Prediction accuracy of different methods.

\begin{tabular}{lcc}
\hline Method & Latitudinal accuracy & Longitudinal accuracy \\
\hline Original-SVM & $86.39 \%$ & $84.20 \%$ \\
PSO-SVM & $92.73 \%$ & $91.64 \%$ \\
\hline
\end{tabular}

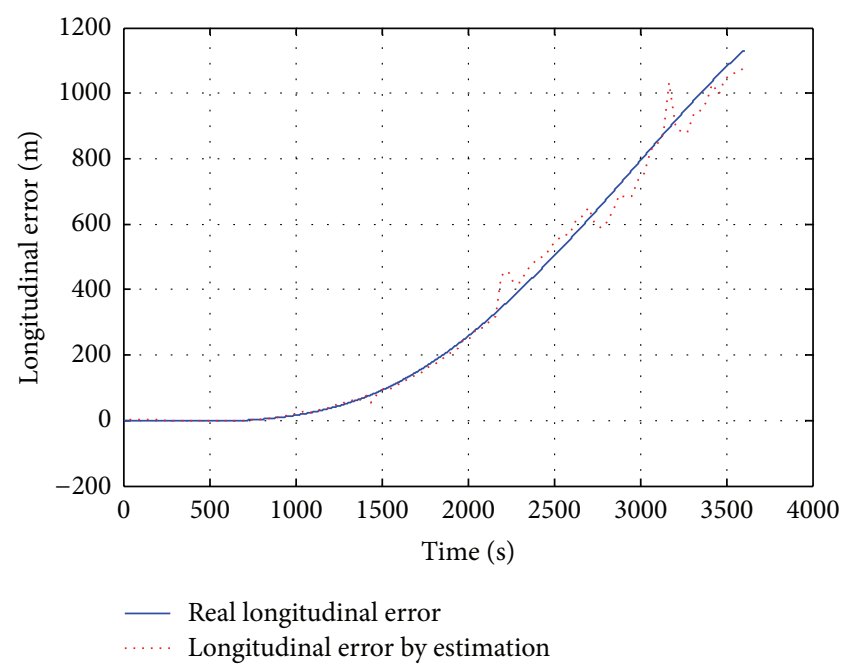

FIGURE 5: Longitudinal error of a strapdown system with known error parameters.

if 100 systems are analyzed, the error model based method takes $2510 \mathrm{~s}$ whereas the SVM-based method only takes $623 \mathrm{~s}$. Compared with analysis method based on error model, the predicting method based on SVM is able to save up to $75 \%$ of calculating time. Therefore, it is effective and efficient to evaluate the navigation errors of SINS.

4.2. Error Prediction of an Entire Flight Process. It is demonstrated that the accuracy of proposed method is satisfying in terms of positioning error prediction. However, it is only available for the prediction with fixed flight time. So, it is necessary to seek a solution to conditions with different flight times.

One strapdown system with known error parameters is analyzed during $3600 \mathrm{~s}$ flight time. 100 models with respect to this system are trained by the given parameters, one model is generated for every $36 \mathrm{~s}$ flight. So, it is achievable to predict the positioning error during the entire flight. The longitudinal error and latitudinal error are seen in Figures 5 and 6 , respectively, which show that at the beginning of the flight when the positioning errors are relatively small, the prediction error is considerably low and can be ignored. The reason is that during the initial period, the training parameters of real positioning errors are small and similar. As a result, SVR-based prediction method tends to generate estimation outputs around that value.

Although several estimating results with big errors with respect to both longitudinal and latitudinal estimation are generated, the overall trends are coherent with real error curves and the overall prediction accuracy is satisfying. Therefore, the proposed method is demonstrated to be 


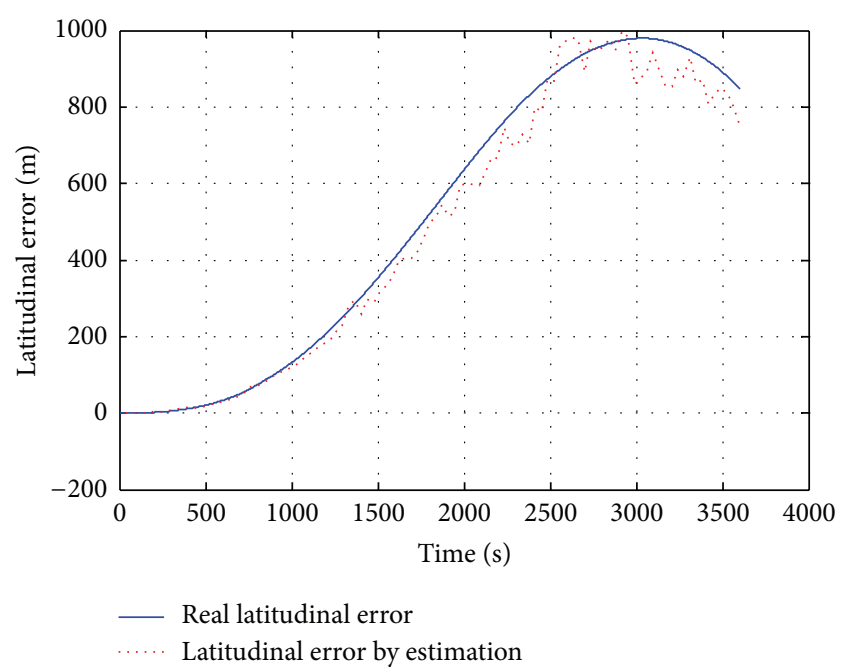

FIGURE 6: Latitudinal error of a strapdown system with known error parameters.

effective for error prediction of an entire flight process with medium accuracy.

\section{Conclusion}

An SVM-based predicting method for SINS positioning errors is proposed, which can be used to assess the performance of navigation systems. Error functions of strapdown navigation systems are established to provide necessary error parameters which are not only used to train SVM model but also utilized to make comparisons with the predicting results of extra systems. RBF is selected to be the kernel function of SVM, and appropriate parameters of SVM are generated by PSO method.

As shown in the numerical verifications, the proposed prediction method is effective in terms of predicting navigation errors of strapdown systems with different error parameters. The accuracy of latitudinal prediction can reach $92.73 \%$, while the accuracy with respect to longitudinal prediction is $91.64 \%$, which is considered to be high enough for application. In addition, this method, compared with error model analysis, can save up to $75 \%$ of calculation time. Finally, the proposed method is demonstrated to be effective for error prediction for an entire flight process, which makes the method more applicable.

Therefore, it enables the researchers to choose appropriate systems for different trajectories or applications by assessing navigation errors efficiently. Since this method is able to evaluate the positioning errors precisely by assessing error parameters of inertial measurement units, it will be useful in terms of error compensation of strapdown navigation systems, which are equipped on different kinds of aircrafts.

\section{References}

[1] Y. Wu, X. Hu, M. Wu, and D. Hu, "Strapdown inertial navigation using dual quaternion algebra: error analysis," IEEE
Transactions on Aerospace and Electronic Systems, vol. 42, no. 1, pp. 259-266, 2006.

[2] Y. Qin, Inertial Navigation, Science Press, Beijing, China, 2009.

[3] X. He, W. Wang, and J. Huang, "Characteristics of gyro error propagation on FOG-SINS," Journal of Chinese Inertial Technology, vol. 15, no. 4, pp. 407-411, 2007.

[4] J. S. Stambaugh, "Propagation and system accuracy impact of major sensor errors on a strapdown aircraft navigator," IEEE Transactions on Aerospace and Electronic Systems, vol. 9, no. 6, pp. 838-846, 1973.

[5] F. Sun, Y.-Y. Ben, and W. Gao, "Application of spiral theory in strapdown inertial navigation algorithm," Systems Engineering and Electronics, vol. 29, no. 9, pp. 1532-1535, 2007.

[6] Y. Hao, J. Gong, W. Gao, and L. Li, "Research on the dynamic error of strapdown inertial navigation system," in Proceedings of the IEEE International Conference on Mechatronics and Automation (ICMA '08), pp. 814-819, August 2008.

[7] F. Gómez-Estern and F. Gordillo, "Error analysis in strapdown INS for aircraft assembly lines," in Proceedings of the 10th International Conference on Control, Automation, Robotics and Vision (ICARCV '08), pp. 184-189, Hanoi, Vietnam, December 2008.

[8] W. Gao, B. Cao, Y. Ben, and B. Xu, "Analysis of gyro's slope drift affecting inertial navigation system error," in Proceedings of the IEEE International Conference on Mechatronics and Automation (ICMA '09), pp. 3757-3762, Changchun, China, August 2009.

[9] H. Musoff and J. H. Murphy, "Study of strapdown navigation attitude algorithms," Journal of Guidance, Control, and Dynamics, vol. 18, no. 2, pp. 287-290, 1995.

[10] J. Wang and H. Gu, "Compensation algorithm of device error for rate strapdown inertial navigation system," in Proceedings of the 1st International Conference on Intelligent Networks and Intelligent Systems (ICINIS '08), pp. 667-670, Wuhan, China, November 2008.

[11] Y.-H. Qiao, Y. Liu, B.-K. Su, and M. Zeng, "Test method for error model coefficients of pendulous integrating gyro accelerometer on centrifuge," Journal of Astronautics, vol. 28, no. 4, pp. 854931, 2007.

[12] T. L. Chen, Estimate at completion for construction projects using evolutionary fuzzy neural inference model [M.S. thesis], Department of Construction Engineering, National Taiwan University of Science and Technology, Taipei, Taiwan, 2008.

[13] J. A. K. Suykens, J. De Brabanter, L. Lukas, and J. Vandewalle, "Weighted least squares support vector machines: robustness and sparce approximation," Neurocomputing, vol. 48, no. 4, pp. 85-105, 2002.

[14] G. L. Plett, "Extended Kalman filtering for battery management systems of LiPB-based HEV battery packs-part 2: modeling and identification," Journal of Power Sources, vol. 134, no. 2, pp. 262-276, 2004.

[15] L. Zhang, " $\mathrm{H}_{\infty}$ estimation for discrete-time piecewise homogeneous Markov jump linear systems," Automatica, vol. 45, no. 11, pp. 2570-2576, 2009.

[16] L. Zhang and E.-K. Boukas, "Stability and stabilization of Markovian jump linear systems with partly unknown transition probabilities," Automatica, vol. 45, no. 2, pp. 463-468, 2009.

[17] L. Zhang and E.-K. Boukas, "Mode-dependent $\mathrm{H}_{\infty}$ filtering for discrete-time Markovian jump linear systems with partly unknown transition probabilities," Automatica, vol. 45, no. 6 , pp. 1462-1467, 2009. 
[18] L. Zhang, P. Shi, E.-K. Boukas, and C. Wang, " $H_{\infty}$ model reduction for uncertain switched linear discrete-time systems," Automatica, vol. 44, no. 11, pp. 2944-2949, 2008.

[19] L. Zhang, E.-K. Boukas, and A. Haidar, "Delay-rangedependent control synthesis for time-delay systems with actuator saturation," Automatica, vol. 44, no. 10, pp. 2691-2695, 2008.

[20] S. R. Gunn, M. Brown, and K. M. Bossley, "Network performance assessment for neurofuzzy data modeling," Advances in Intelligent Data Analysis Reasoning About Data, vol. 1280, pp. 313-323, 1997.

[21] T. Hansen and C.-J. Wang, "Support vector based battery state of charge estimator," Journal of Power Sources, vol. 141, no. 2, pp. 351-358, 2005.

[22] J. Nong, "Parameters selection and noise estimation of SVM regression," in Proceedings of the 5th International Joint Conference on Computational Sciences and Optimization, pp. 379-381, Harbin, China, 2012.

[23] K.-W. Yan, "Study on the forecast of air passenger flow based on SVM regression algorithm," in Proceedings of the 1st International Workshop on Database Technology and Applications (DBTA '09), pp. 325-328, Wuhan, China, April 2009.

[24] P. G. Savage, "Strapdown inertial navigation integration algorithm design - part 1: attitude algorithms," Journal of Guidance, Control, and Dynamics, vol. 21, no. 1, pp. 19-28, 1998.

[25] T. Hansen and C.-J. Wang, "Support vector based battery state of charge estimator," Journal of Power Sources, vol. 141, no. 2, pp. 351-358, 2005.

[26] J. C. Alvarez Anton, P. J. Garcia Nieto, C. Blanco Viejo, and J. A. Vilan Vilan, "Support vector machines used to estimate the battery state of charge," IEEE Transactions on Power Electronics, vol. 28, no. 12, pp. 5919-5926, 2013.

[27] I. Steinwart, D. Hush, and C. Scovel, "An explicit description of the reproducing Kernel Hilbert spaces of Gaussian RBF kernels," IEEE Transactions on Information Theory, vol. 52, no. 10, pp. 4635-4643, 2006.

[28] Y.-J. Oyang, S.-C. Hwang, Y.-Y. Ou, C.-Y. Chen, and Z.-W. Chen, "Data classification with radial basis function networks based on a novel kernel density estimation algorithm," IEEE Transactions on Neural Networks, vol. 16, no. 1, pp. 225-236, 2005.

[29] G. F. Smits and E. M. Jordaan, "Improved SVM regression using mixtures of kernels," in Proceedings of the International Joint Conference on Neural Networks (IJCNN '02), pp. 2785-2790, Honolulu, Hawaii, USA, May 2002.

[30] H. Wang, Z. Hu, M. Hu, and Z. Zhang, "Short-term prediction of wind farm power based on PSO-SVM," in Proceedings of the Power and Energy Engineering Conference, pp. 1-4, Shanghai, China, 2012.

[31] Y. Bazi and F. Melgani, "Semisupervised PSO-SVM regression for biophysical parameter estimation," IEEE Transactions on Geoscience and Remote Sensing, vol. 45, no. 6, pp. 1887-1895, 2007.

[32] M. Pastorino and A. Randazzo, "The SVM-based smart antenna for estimation of the directions of arrival of electromagnetic waves," IEEE Transactions on Instrumentation and Measurement, vol. 55, no. 6, pp. 1918-1925, 2006. 


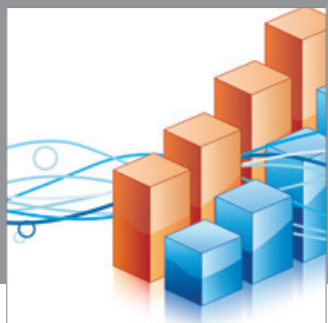

Advances in

Operations Research

mansans

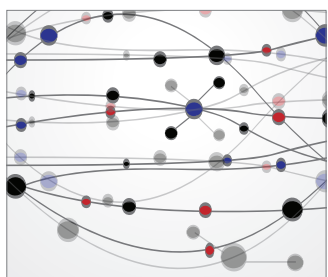

The Scientific World Journal
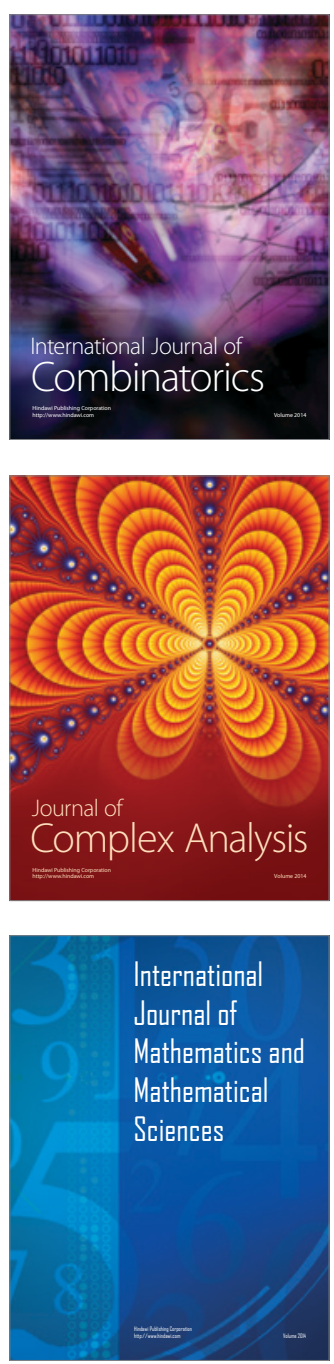
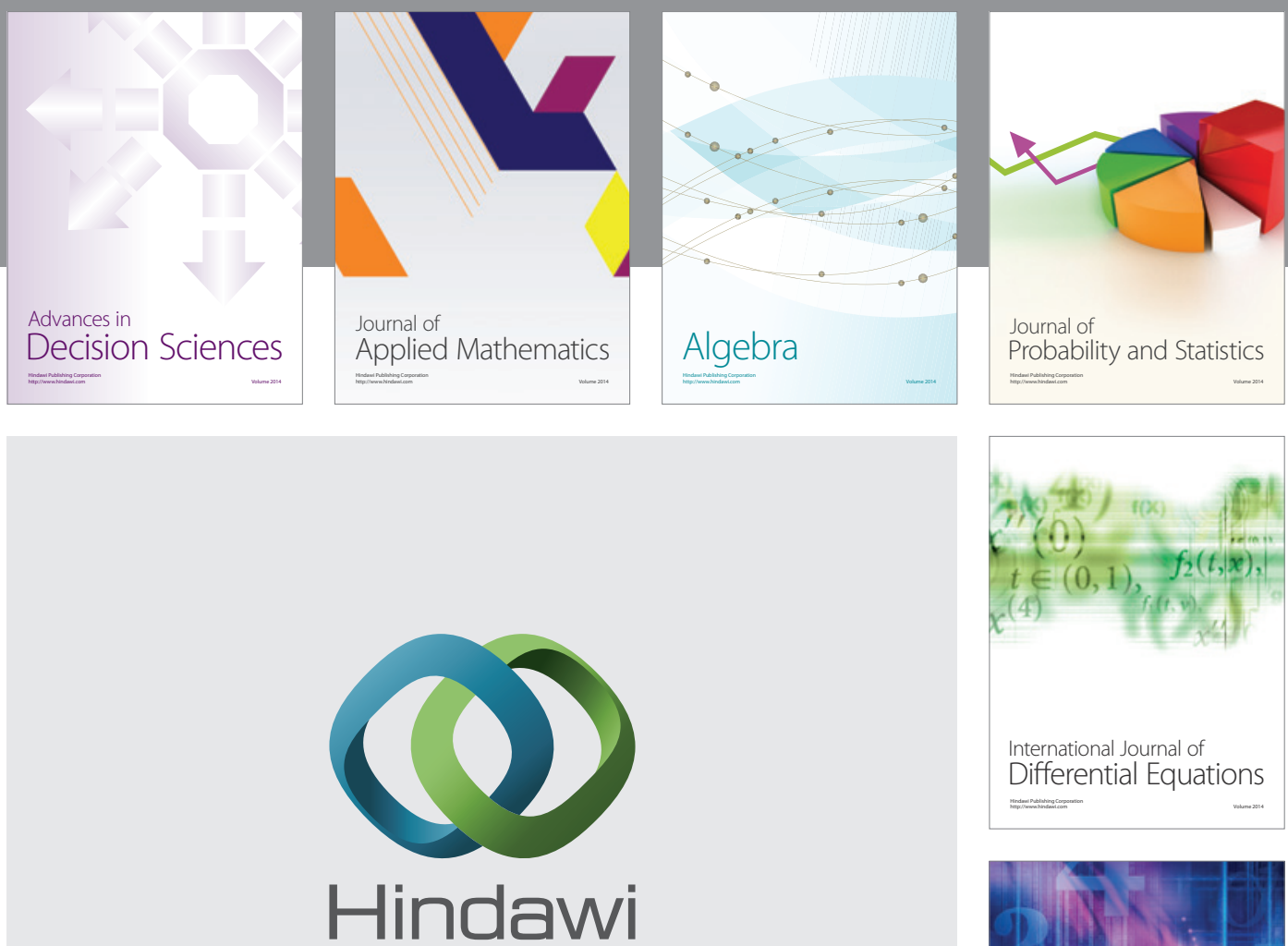

Submit your manuscripts at http://www.hindawi.com
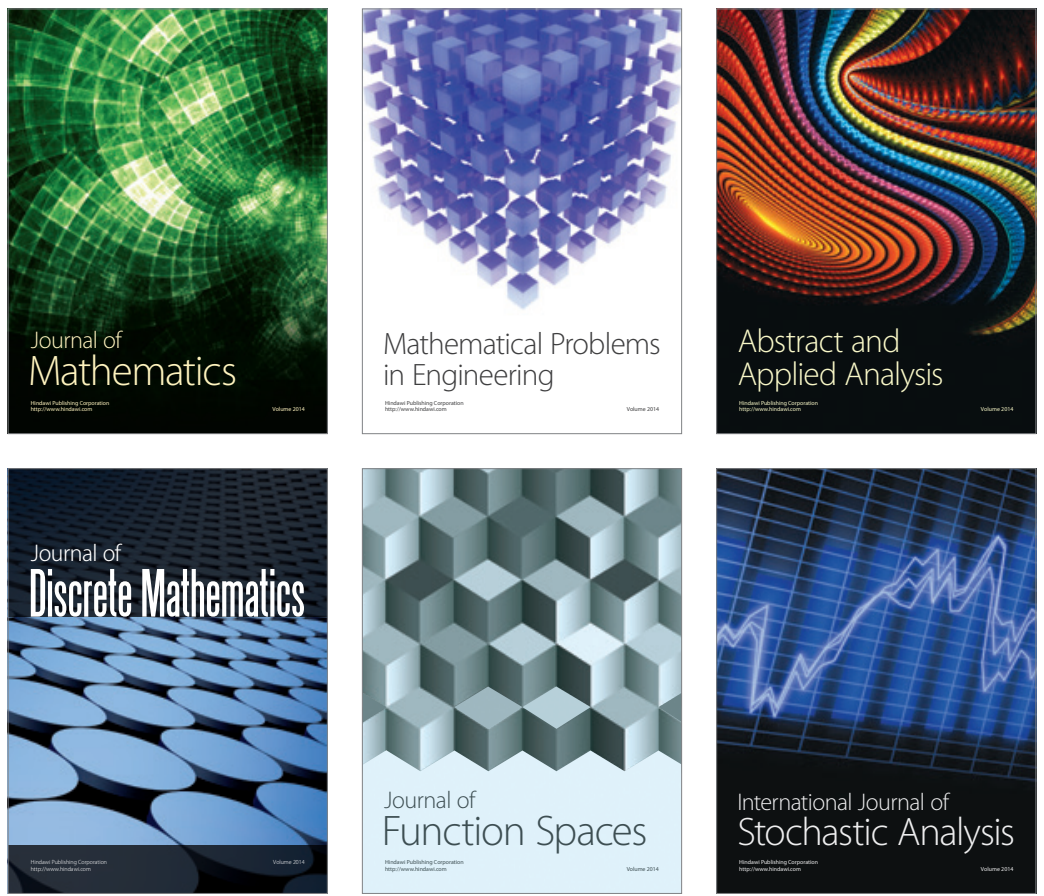

Journal of

Function Spaces

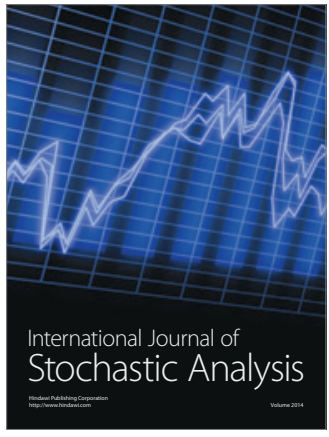

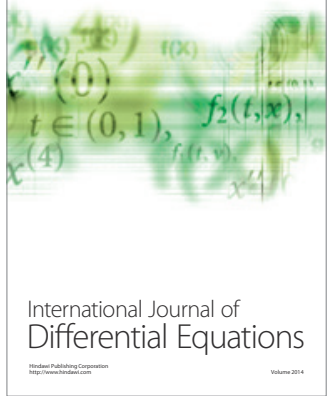
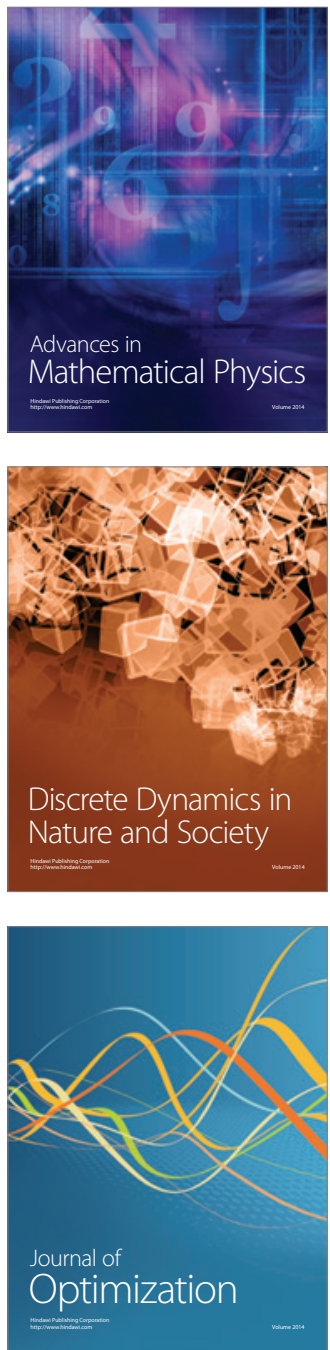